\title{
Minimum loss multiplicative routing metrics for wireless mesh networks
}

\author{
Diego Passos • Célio Vinicius N. de Albuquerque • \\ Miguel Elias M. Campista • Luís Henrique M.K. Costa • \\ Otto Carlos M.B. Duarte
}

Received: 26 August 2010 / Accepted: 29 November 2010 / Published online: 30 December 2010

(C) The Brazilian Computer Society 2010

\begin{abstract}
Wireless mesh networks are low-cost self-configurable multihop networks. This work presents a study about the main routing metrics used in this kind of network, pointing out their virtues and limitations. It also proposes, implements, and analyzes alternative multiplicative metrics. To evaluate the performance of the proposed metrics, comparative measurements over real mesh testbeds were conducted. A case study of a production mesh network using a multiplicative routing metric is also evaluated. Results show that, in various scenarios, the network performance with the proposed multiplicative routing metrics has been improved in terms of routing stability, packet loss rate, end-to-end delay, and throughput.
\end{abstract}

Keywords Wireless mesh networks · Routing metrics · Routing protocols $\cdot$ Multihop networks

\footnotetext{
D. Passos · C.V.N. de Albuquerque ( $\varangle$ )

Instituto de Computação, Universidade Federal Fluminense,

Niterói, RJ, Brazil

e-mail: celio@ic.uff.br

D. Passos

e-mail: dpassos@ic.uff.br

M.E.M. Campista - L.H.M.K. Costa · O.C.M.B. Duarte

GTA/COPPE/POLI/UFRJ, Universidade Federal

do Rio de Janeiro, Rio de Janeiro, RJ, Brazil

M.E.M. Campista

e-mail: miguel@gta.ufrj.br

L.H.M.K. Costa

e-mail: luish@gta.ufrj.br

O.C.M.B. Duarte

e-mail: otto@gta.ufrj.br
}

\section{Introduction}

Ad hoc networks are wireless multihop networks with mobility support and self-configuring features. These networks are opposed to structured wireless ones, in which fixed access points relay all client communications. In an ad hoc network, there is no need for such infrastructure, as the transmission between two clients is performed either in a direct way, or through multiple hops. In the last case, intermediate nodes act as routers forwarding packets toward their final destination node.

In a multihop wireless scenario, a new concept of network emerges: the wireless mesh networks (WMNs). In wireless mesh networks, there is a subset of stationary nodes, called mesh routers, which are in charge of acting as routers for potentially mobile client nodes. Communication between the WMN and other networks (e.g., the Internet) is performed by border mesh routers named mesh gateways. In WMNs, each mesh router maintains links to other mesh routers, such that the topology obtained is a mesh of wireless links [1]. One major advantage of this approach, when compared to ad hoc networks, is the fact that there is a specific set of nodes, namely the mesh routers, responsible for forwarding packets. Since each node has several alternative paths to the same destination, routing becomes a non-trivial task in this type of network.

The organic growth of ad hoc networks is preserved in WMNs, as adding a new mesh router to the network increases the number of alternative paths. Although mobility in wireless mesh networks is reduced because of the static mesh routers, the network remains dynamic. User mobility and wireless medium dynamics are still important issues to be considered because they can lead to frequent route breakages. Wireless transmissions still have to face fast link quality changes and the network has to deal with addition of new 
mesh nodes in an autonomous fashion. Given these similarities with ad hoc networks, and moreover the transmission in multiple hops, it is natural to use ad hoc routing protocols in WMNs. Nevertheless, several peculiarities, such as the frequent communication to and from mesh gateways, make the development of mesh routing protocols desirable [5].

The goal of this work is to define the characteristics of a wireless mesh network and use them to classify the quality of a route in this network. In the past, researchers have disregarded the study of multiplicative composition functions since they fail to account for intraflow interference [8, 11]. Recent works [5, 7, 12, 17, 23, 24] refer to the Minimum Loss (ML) multiplicative metric as an alternative to traditional additive metrics, however, no comprehensive study of this metric has been performed so far. This work evaluates the performance of ML in a real WMN in production since March 2006. Network simulations are also performed to corroborate the ML performance in other scenarios. As argued in Sect. 5, this work claims based on its results that multiplicative composition functions can provide routing metrics with low packet loss rate, low delay, and throughput comparable to the obtained with additive metrics and, therefore, deserve to be investigated.

This work is organized as follows. Section 2 describes the main characteristics of wireless mesh networks, as well as the main challenges of designing a mesh routing protocol. A model for WMNs is introduced in Sect. 3. In Sect. 4, a description of routing metrics used in WMNs is presented. In Sect. 5 a set of multiplicative metrics is proposed. Section 6 depicts the evaluation testbeds and in Sect. 7 performance results are presented. A case study of a production mesh network using a multiplicative routing metric is shown in Sect. 8. Finally, Sect. 9 presents some concluding remarks.

\section{Wireless Mesh Networks}

A WMN is typically composed of a set of nodes which can be divided in two types: mesh routers and mesh clients [1]. Mesh routers are basically static, whereas mesh clients can be mobile, as in ad hoc networks.

Mesh routers form the network backbone, which can be connected to other networks through specific nodes acting as gateways. On the other hand, clients may use the mesh network through a wireless interface, or they can be directly connected to a mesh router using other technologies such as Ethernet. Figure 1 illustrates an example of mesh topology.

Some interesting features of WMNs include: low cost, self-configuration, and fault-tolerance [4]. The network must be able to adapt to changes caused by the addition, removal, or failure of certain nodes. More specifically, the deployment of a wireless mesh network can be done gradually. Mesh routers can be installed as needed and as resources

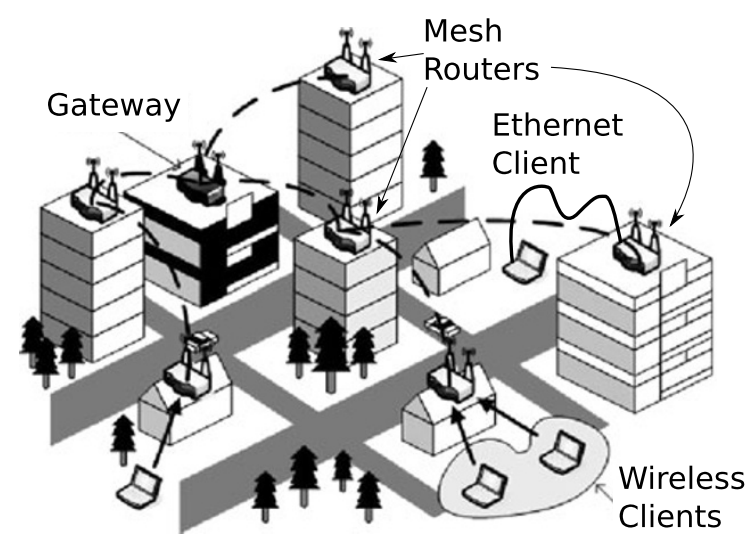

Fig. 1 Example of a wireless mesh network topology

become available. Hence, the initial investment can be considerably low. Besides, only a reduced set of nodes need to be directly connected to a cabled infrastructure, further contributing to the reduced costs in infrastructure. Regarding fault-tolerance, increasing the number of mesh routers in the backbone also increases the probability of finding alternative paths. Moreover, there is no central point, limiting the damage caused by a router failure. Multiple gateways can be deployed as well.

For all the above mentioned qualities, wireless mesh networks are considered a very promising technology [1], especially in environments where deploying a more complex network infrastructure is unfeasible. Although it has been shown that the throughput of a WMN decreases rapidly with the increase in the number of hops [3], there are known techniques to avoid this performance loss, such as multihoming, i.e., the deployment of multiple gateways [10].

\subsection{Difficulties in finding the best route}

At the routing layer, the development of new protocols and metrics is fundamental. Though possible, the use of ad hoc routing protocols in mesh networks can bring some inconveniences. One of these inconveniences is the large amount of control packets used by ad hoc protocols. Because of the high dynamics of ad hoc networks, it becomes necessary to either constantly evaluate the network topology, or to flood the network with route discovery control packets in an ondemand fashion. On the other hand, in wireless mesh networks the backbone topology is static and in many cases most of the traffic comes from and goes to mesh gateways. Hence, a good routing protocol for mesh networks must be stable and must use network resources efficiently.

Regarding routing metrics, even in ad hoc networks the existent solutions are not optimal. One of the reasons is the difficulty to model this kind of system, given factors such as: 
Table 1 Statistic from the quality of each link in a wireless mesh network during one day

\begin{tabular}{llrrr}
\hline Link & Minimum & Maximum & Average & $\begin{array}{r}\text { Standard } \\
\text { deviation }\end{array}$ \\
\hline L1 & 1.05 & 71.30 & 9.40 & 7.03 \\
L2 & 1.00 & 1.97 & 1.06 & 0.07 \\
L3 & 1.00 & 51.00 & 1.12 & 2.07 \\
L4 & 1.00 & 53.12 & 10.09 & 8.02 \\
L5 & 1.00 & 451.56 & 90.91 & 72.11 \\
L6 & 1.00 & 2.21 & 1.07 & 0.09 \\
L7 & 1.00 & 13.42 & 1.13 & 0.17 \\
L8 & 1.00 & 104.04 & 2.40 & 4.08 \\
L9 & 1.00 & 451.56 & 199.60 & 180.58 \\
L10 & 1.00 & 1.32 & 1.02 & 0.03 \\
L11 & 1.00 & 1.39 & 1.07 & 0.06 \\
L12 & 1.00 & 1.24 & 1.01 & 0.03 \\
L13 & 1.00 & 2.28 & 1.06 & 0.09 \\
L14 & 1.00 & 68.45 & 1.05 & 0.42 \\
L15 & 1.00 & 30.44 & 1.04 & 0.19 \\
L16 & 1.00 & 451.56 & 1.20 & 4.19 \\
L17 & 1.00 & 51.00 & 6.10 & 3.54 \\
L18 & 1.00 & 141.67 & 1.10 & 2.16 \\
L19 & 1.00 & 106.25 & 2.25 & 2.17 \\
L20 & 1.05 & 425.00 & 8.21 & 6.58 \\
\hline & & & & \\
& & & & \\
\hline
\end{tabular}

- The variability of the transmission rates: wireless networks usually support various modulations and code rates resulting on multiple transmission rates that nodes can dynamically alter. Therefore, the capacity from each node for transmitting packets may vary in time.

- And the sensibility to external interferences: packet losses and significant increase in latency may happen even when routers are not overloaded, because of transmission errors.

Another consequence of the last item is the high variability of the quality in wireless links. To illustrate it, Table 1 shows values obtained through the monitoring of a real wireless mesh network-described in Sect. 8-during 24 hours. Each row presents the average, standard deviation, maximum and minimum values for the ETX (Expected Transmission Count) metric, a possible measurement for the quality of the network links.

The ETX metric is explained in detail on Sect. 4. ETX values can vary from 1 to $\infty$, where 1 represents a perfect link, whereas values above 10 represent a poor link. We can see that even very poor-quality links with average of 90 or more, such as link L5, at some point behaved as a perfect link. Therefore, it is clearly hard to make a coherent evaluation from links in this kind of network.

The evaluation of a complete route is even more challenging than only evaluating a link. When a node sends data over a wireless link, it reaches and possibly interferes with all its neighbors, because the medium is shared. Thus, it is not sufficient to only evaluate the link qualities individually, but it is necessary to consider the effects of transmissions along a path and its vicinity.

All those factors explain the difficulties of routing in wireless mesh networks and, therefore, justify the motivations for this work.

\section{Network model}

We model a wireless mesh network as a weighted directed graph $G=(V, E)$ where $V$ is the vertex set and $E$ is the edge set. Vertices represent network nodes and edges represent links between nodes. Considering $u$ and $v$ two nodes in $V$, if there is a link from $u$ to $v$, then $u v \in E$. There is a weight $w_{u v} \in \Re_{+}$associated with each link in the network. In the routing context, each edge weight represents the routing metric of the link.

A path $p$ is a sequence of distinct nodes in which any consecutive pair is connected by a link. Therefore, $p=\left\langle v_{1}, v_{2}, \ldots, v_{n-1}, v_{n}\right\rangle$, where $n$ is the length of $p$, $\left\{v_{1}, v_{2}, \ldots, v_{n-1}, v_{n}\right\} \subseteq V$, and $\left\{v_{1} v_{2}, \ldots, v_{n-1} v_{n}\right\} \subseteq E$. The path cost is defined by a composition function $f$ that maps the weights of the links in $p$ to a nonnegative real number. Hence, $f: w_{v_{1} v_{2}}, \ldots, w_{v_{n-1} v_{n}} \rightarrow \Re_{+}$.

The routing protocol defines the routing metric and the algorithm to compute the best path between any two nodes in the network. The routing metric is determined according to specific quality requirements the protocol aims at optimizing. For example, a typical routing metric is the available bandwidth of a link. The best path, in this case, is the path with the maximum available bandwidth considering all links in $E$. We define, thus, the best path between any two nodes as the one that optimizes the path cost taking into account the routing metric and the composition function used.

The composition function $f$ used to compute path costs can follow three basic approaches: additive, multiplicative, or concave [9]. The additive composition function sums link weights along the path $p$. The multiplicative multiplies those link weights. The concave composition function chooses the path that has among its links the one with the minimum (or maximum, depending on the metric) weight.

In wireless networks, links are prone to transmission failures. A transmission is considered successful in IEEE 802.11 if a frame is sent and its respective acknowledgment is received back. Thus, the data transmission on a link depends on its forward and backward delivery rates. We denote the transmission success probability on a link $u v$ as $P_{u v}=d_{f} \times d_{b}$, where $d_{f}$ and $d_{b}$ are the delivery rates on forward and backward directions, respectively. Note that $P_{u v}$ considers delivery rates independent variables. One way 
to compute delivery rates is to have each node periodically broadcast a probe in the network. The delivery rate is the number of received probes over the maximum possible number in a sliding window. It is worth mentioning that nodes cannot measure forward delivery rates. Thus, each node includes on its probes information about the backward delivery rate measured from each node within its transmission range.

\section{Routing metrics}

This section presents typical metrics used by protocols that implement additive and multiplicative composition functions. For a deeper discussion on the metrics presented here and others, please refer to [5, 22].

\subsection{Additive metrics}

Many routing protocols use additive composition functions. In this approach, the path cost from any pair of nodes in the network is computed by a composition function such as $\sum_{u v \in p} w_{u v}$, where $u v$ are the links of path $p$. The hop count is a typical additive metric. It simply assigns the weight 1 for connected links and 0 , otherwise. Therefore, the best path between any source-destination pair is the one with the lowest number of hops. The hop count, however, leads to poor performance because it likely chooses links with high loss rates or low throughput [11]. Minimizing the number of hops results in paths composed of links between distant nodes. These links frequently have low SNR or reduced transmission rates.

The Expected Transmission Count (ETX) metric [11] improves the network throughput by reducing the total number of transmissions per link. Considering $P_{u v}$ the success probability of a frame transmission on link $u v$, the average number of transmission attempts to get one successful transmission is $\frac{1}{P_{u v}}$. ETX is the inverse of the success probability of a frame transmission on a link. De Couto et al. [11] show that the network performance using ETX increases considerably as compared with hop count.

A shortcoming of ETX arises from the success probability overestimation by broadcasting probes. Broadcast frames are transmitted at the network basic rate, which is the lowest and more robust rate used in the network, according to IEEE 802.11 standard. This is a desirable characteristic to achieve the maximum possible number of nodes. The network basic rate varies according to the IEEE 802.11 standard in use, namely amendments $\mathrm{a} / \mathrm{b} / \mathrm{g}$, and can be as low as $1 \mathrm{Mb} / \mathrm{s}$. The success probabilities may be overestimated because data communications are typically performed at higher rates, whereas ETX delivery rates are estimated at lower rates.
The Expected Transmission Time (ETT) [13, 15] metric is an extension of the ETX metric, which tries to solve the problem of overestimated success probability. ETT considers the transmission rate used on the link to perform a more accurate success probability estimation. The idea is to obtain a value that reflects the average link delay considering possible retransmissions. This can be obtained multiplying the ETX of a link by the time needed to transmit a single frame, $t$. The time $t$ is computed considering the packet size and data transmission rate of the link. Therefore, $\mathrm{ETT}=\mathrm{ETX} \times t=\mathrm{ETX} \times \frac{S}{B}$, where $S$ is a typical frame size and $B$ is the data transmission rate on the link. Retrieving the current data transmission rate, however, is challenging because many wireless cards do not provide such information through the driver. Draves et al. [13] use the packet pair probing technique to estimate the data transmission rate per link. Bicket et al. [3], on the other hand, perform broadcast transmissions at different modulations to check the one that offers the best throughput. The former technique requires unicast transmissions to each neighbor, whereas the latter requires frequent changes to the broadcast transmission rate, a feature that is not supported by all wireless card drivers.

\subsection{Multiplicative metrics}

A number of wireless mesh network routing protocols rely on the metrics discussed in Sect. 4.1, which use additive composition functions. Multiplicative and concave metrics are rarely used by WMN routing protocols. In this work, we evaluate the performance of multiplicative approaches. Using multiplicative metrics, the path cost from any pair of nodes in the network is computed using a composition function such as $\prod_{u v \in p} w_{u v}$, where $u v$ are the links of path $p$. It is worth mentioning that typical routing algorithms use additive composition functions. Hence, employing multiplicative metrics imply modifications on Dijkstra or BellmanFord.

De Couto et al. [11] claim that multiplicative composition functions fail to account for multihop intraflow interference. A protocol using multiplicative functions would pick a path composed of two perfect links instead of a one-hop path with $90 \%$ success probability. The path chosen with a multiplicative function would, therefore, result on an increased number of medium accesses compared with the additive approach. This example gives a picture of a potential shortcoming of the multiplicative approach. Nevertheless, the choice between a path composed of two perfect links and an alternative one-hop path with $51 \%$ success probability is no longer that obvious. In practice, we observe that links with lower success probability may experience more instability, which was not considered by De Couto et al. [11]. Figure 2 plots the standard deviation of the ETX metric against 


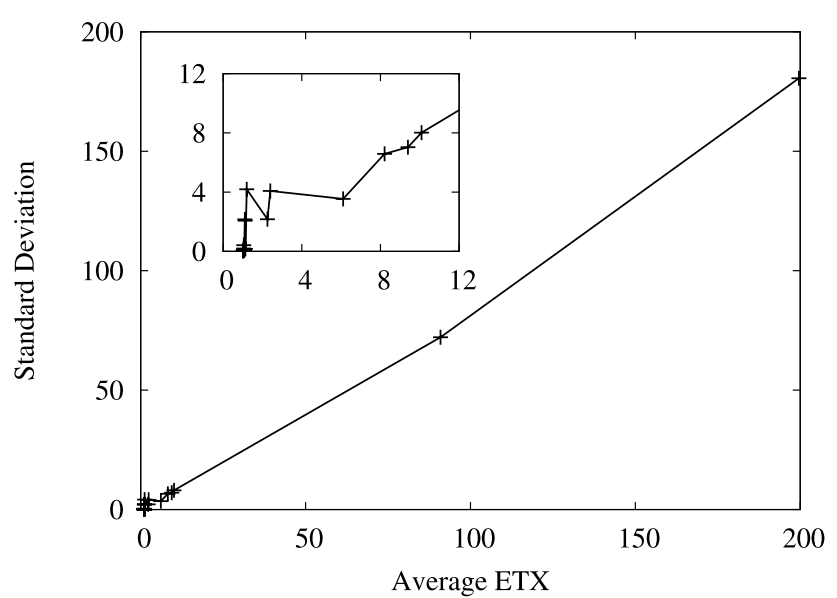

Fig. 2 Variation of ETX of the links in Table 1

its average value (Table 1). The plot reveals that the variance of each link ETX increases with the average value almost linearly. Multiplicative metrics may not consider multihop interference, but additive metrics may choose unstable links. Rather than choosing consecutive perfect links, additive metrics choose fewer links but with higher average ETX values. The indirect consequence is the use of more unstable links since instability and average ETX values augment proportionally. Link instability leads to poor network performance and must be avoided in operational networks. Koksal and Balakrishnan [19] propose the mETX (modified ETX) metric, which considers intrapacket link instability and does not only consider average values. This technique is only applicable to control packets that follow a known bit pattern, since it needs to compute the amount of wrong bits. However, its implementation is not trivial in practice, since control packets typically have long interarrival periods and each node must know if a received corrupted packet is a control packet in order to properly compute this metric.

Paths would become longer in number of links if unstable links were avoided. The ETX metric, however, is resistant to the addition of a new link. This behavior is illustrated in Fig. 3. Let $r$ be the number of received probes in a window of time. Considering that a probe is sent per unit of time and that the sliding window size contains $w$ units of time, then $d_{f}=d_{b}=\frac{r}{w}$ and ETX $=\frac{1}{r^{2} / w^{2}}$. An increment on ETX caused by a single lost probe in function of $r$ is given by:

$\delta=\frac{w^{2}}{r^{2}(r-1)}$.

Figure 3 shows a few values for sliding window sizes $(w)$ of $10,20,50$, and 100 . This increment is quite low for relatively high values of $r$. Only for lower values of $r$, the function assumes values close to or higher than 1 , the minimum weight of a link for this metric. Therefore, ETX requires many losses for a longer path to be chosen over a

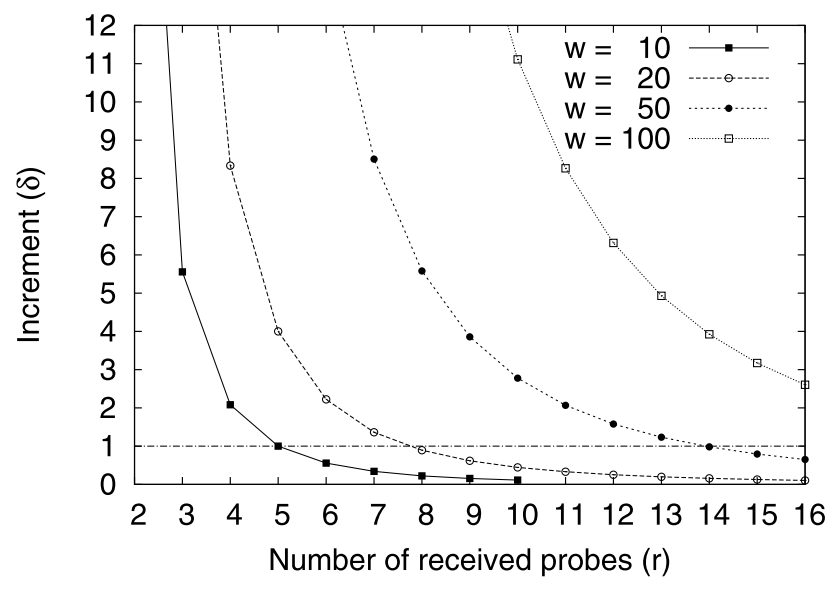

Fig. 3 Values of increment for different ETX window sizes $(w)$

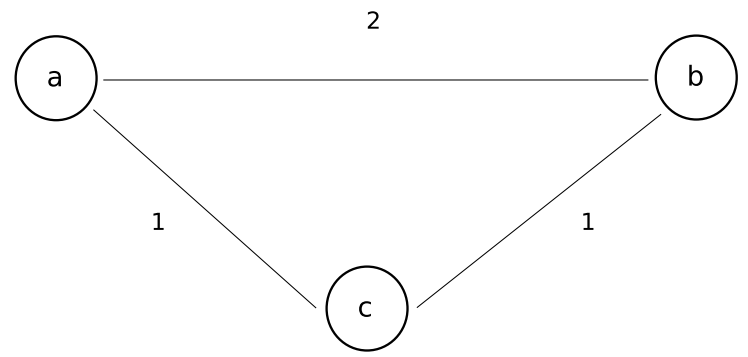

Fig. 4 Example of topology for which ETX would choose a high loss path, instead of more stable links

shorter path. This characteristic leads to paths including unstable links. Figure 4 illustrates this behavior with a small topology example. In the example, node $a$ has two possible paths to reach node $b$ : a direct route or an alternative, using node $c$ as a relay. The cost on each edge represents the ETX for the link. Therefore, the ETX metric would evaluate both paths as being equally good (both have a total ETX of 2). In this situation, the direct route would be chosen due to its lower number of hops. However, this choice would result in the usage of link with a 50\% loss rate at the link-layer, while the alternative path has a $0 \%$ estimated loss rate.

All metrics presented in this section have the goal of optimizing a given network performance parameter (e.g., endto-end delay or packet loss rate) considering a single packet. In other words, these metrics do not model the behavior of the network in case there are concurrent flows or bursts of packets. Due to this reason, following the methodology adopted in previous papers $[5,11,15]$, we do not consider multiple flows in our analysis, although it would be interesting to do such an evaluation in the future. 


\section{Proposed multiplicative routing metrics}

In this work, we propose and investigate two routing metrics to evaluate multiplicative composition functions. The main goal of these metrics is to minimize the path loss rate.

\subsection{The Minimum Loss metric}

The Minimum Loss (ML) metric is based on the intuition that computing the path that introduces the minimum loss rate requires multiplying the success probability of each link along the path. This is a rough estimate once the product of success probabilities assumes independent events. In practice, though, we see that this simplification is in many cases reasonable according to measurements on our test network.

In ML, the weight associated with each link $u v$ is the probability $P_{u v}$ that a frame is successfully transmitted from node $u$ to node $v$. The best path between two nodes is the one with the greatest end-to-end success probability. Assuming independent success probabilities, the best path between two nodes is the one with the greatest product of individual link weights. We define the ML metric of a path composed of $n$ links as:

$\mathrm{ML}=\prod_{u v \in p} P_{u v}$

ML chooses paths with a higher number of links, where each link is probably shorter and presents a better success probability compared to links chosen by ETX. The ML metric, however, presents a shortcoming. It cannot differentiate paths composed of two perfect links from paths composed of three perfect links. Therefore, there are no guarantees that ML can find the shortest path with the minimum possible number of links. Section 5.2 copes with this issue.

\subsection{The ML metric with an additive cost}

Routing metrics define the range of link weights. This range depends on the shortest-path algorithm and on the composition function used. In other words, the addition of a new link to a route should change the cost of the path to guarantee that the shortest-path algorithm indeed finds the optimal solution. For instance, the Dijkstra algorithm does not handle negative link weights. Problems such as excessively long paths or infinite loops may occur.

The proposal of the ML metric allows the use of the value 1 as a valid link weight. Nevertheless, this value is the identity element of multiplication operations and it does not change the cost of a path. Based on the ML proposal, there are two alternatives to deal with this case. The first option is to rescale the interval of possible values. For example, instead of using the interval $w_{u v} \in[0,1]$, one could use $w_{u v} \in[0,0.5]$. In this case, the link weight is equal to the success probability divided by 2 , and the product of the weights of two perfect links (links with loss probability equal to 0 ) is lower than the greatest possible value. The second alternative is to manipulate the ML metric to avoid unitary link weights. In this work, we propose a metric which adopts the last option.

We define the end-to-end Minimum Loss with Additive Cost (MLAC) metric from a path $p$ :

$\mathrm{MLAC}=\prod_{u v \in p}\left(\frac{1}{\mathrm{ETX}_{u v}+\lambda}\right)$,

where the adjustable parameter $\lambda$ is a non-negative constant and ETX is the respective metric of link $u v$ in the path $p$. We avoid the unitary link weight as long as $\lambda>0$. The constant $\lambda$ added to ETX represents a constant used to avoid paths with a higher number of hops. In Sect. 7, we show the impact of $\lambda$ on network performance.

\section{Performance evaluation setup}

We evaluate the performance of the proposed metrics via experimental analysis and simulations. Our experimental tests were performed using the available structure of the ReMesh project [23]. Three different topologies were used, one outdoor and two indoor. The three topologies are composed of Linksys WRT54G routers with a customized Linux-based firmware called OpenWRT [21]. In addition to our experimental tests, we also provide simulations using the ns-2 simulator [16] (version 2.29). The goal is to verify if the experimental results are confirmed in a controlled environment. We consider this second evaluation important because experimental analysis is prone to frequent changes in network conditions. To accomplish that, we reproduce as close as possible our experimental environment in the simulator.

In this work, we evaluate the performance of the proposed multiplicative metrics using the OLSR (Optimized Link-State Routing) protocol [6]. OLSR is a link-state-based protocol widely used in current wireless mesh networks. OLSR implements the Dijkstra algorithm for route computation. Both in our experimental analysis and in our simulations, some modifications to the available implementations were required. These modifications were basically related to changes to the composition function used by Dijkstra algorithm, which was inherently additive. The open source Linux-based firmware for wireless routers [20] and the C++ code of the ns-2 simulator [25] made the modifications on the OLSR code quite simple in both cases. The ETX metric, however, is already implemented as the default option of the firmware, but it is not implemented in the ns- 2 code. Hence, we needed to change the simulator code to implement also the ETX metric. In addition, we used an improved 


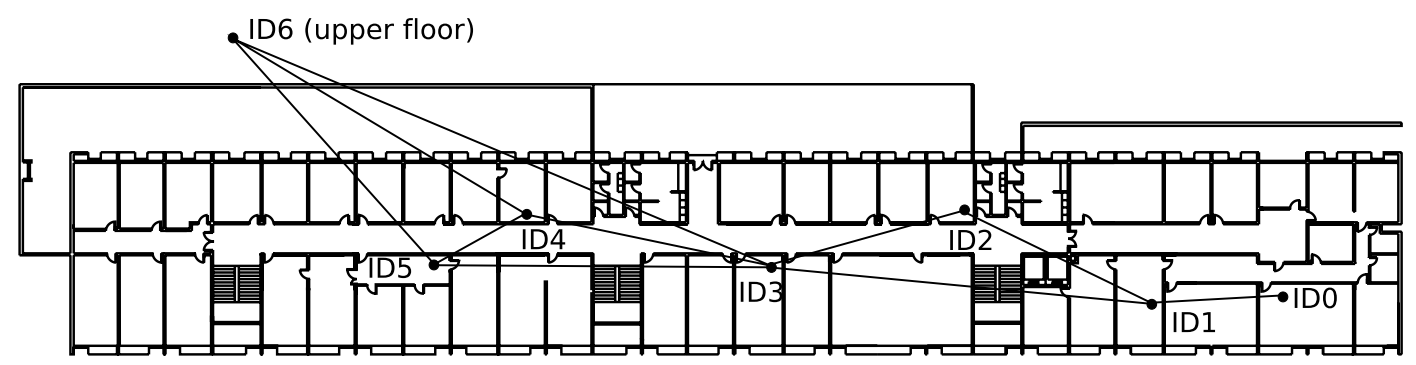

Fig. 5 Indoor network A

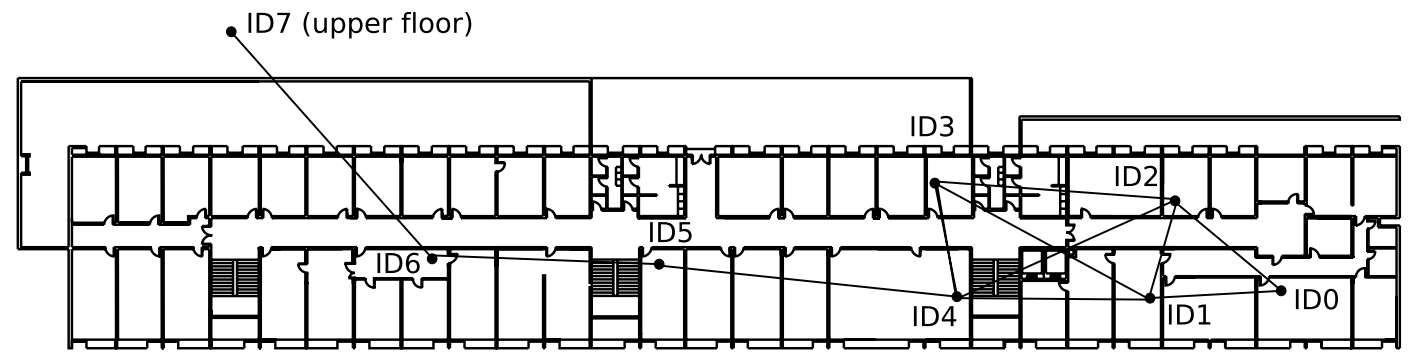

Fig. 6 Indoor network B

Table 2 Parameters used for the Shadowing propagation model

\begin{tabular}{ll}
\hline Parameter & Value \\
\hline Path loss exponent & 2.15 \\
Standard deviation & 1.4 \\
Reference distance & 0.5 \\
\hline
\end{tabular}

PHY-layer and IEEE 802.11 modules of ns- 2 by using modifications proposed by the ns-miracle implementation [2]. We adopted Shadowing as the propagation model for the simulations. Before we start the actual simulations, the parameters of the model were adjusted so that the characteristics of the network links were close to those of the real testbed. Table 2 shows the values obtained after this adjust. Nevertheless, since in the actual testbed the propagation environment is not homogeneous, it is not possible to fully reproduce the scenario using Shadowing. Therefore, the absolute values of the results obtained through simulation and real experimentation may differ. Due to this reason, in our analysis we do not compare the absolute values, but the performance trends of simulated and real environments.

We consider two different indoor topologies in our experimental analysis. The same topologies are used in our simulations. The indoor evaluation is performed in two different networks built inside one of the Engineering buildings of the Fluminense Federal University. The nodes are placed in rooms and laboratories along two floors of the building. Figure 5 shows the first topology, Indoor network A, which is composed of seven routers. The second topology, Indoor network $B$, is illustrated on Fig. 6 .
Indoor networks do not experience the same weather variations faced by the outdoor network. Indoor communications, however, can be highly attenuated by obstacles, such as walls, elevators, and walking people. In addition, these networks can be affected more severely by other networks at the same operating channel.

\section{Results}

In the following subsections, the performance results obtained in each topology are presented and discussed.

\subsection{ML metric analysis}

We conduct comparative tests using ML and ETX metrics. Our evaluation used three performance metrics: packet loss, round trip time (RTT), and throughput. These tests were conducted in the indoor topology A.

\subsubsection{Packet loss}

The packet loss tests were performed by a script sending a set of pings. Pings are 84-byte packets (considering headers) transmitted per second. In our experiments, we used a Linux tool whereas in the simulations, we used a module to generate ping messages available in the ns- 2 code. Figure 7(a) shows the results obtained.

In the indoor topology A, the test was performed during a 12-hour period. At each second, a ping message is sent from one network extreme to the other (i.e., from node 0 to 


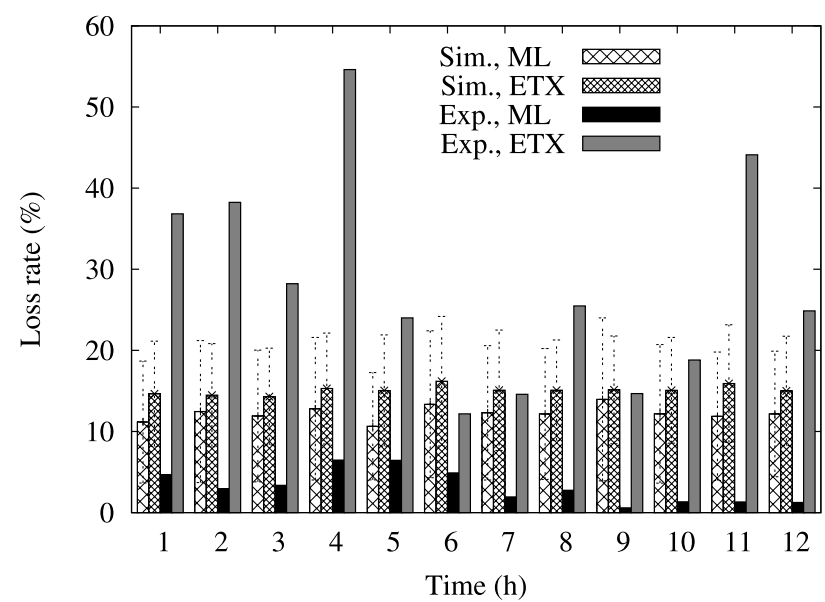

(a) Packet loss rate.

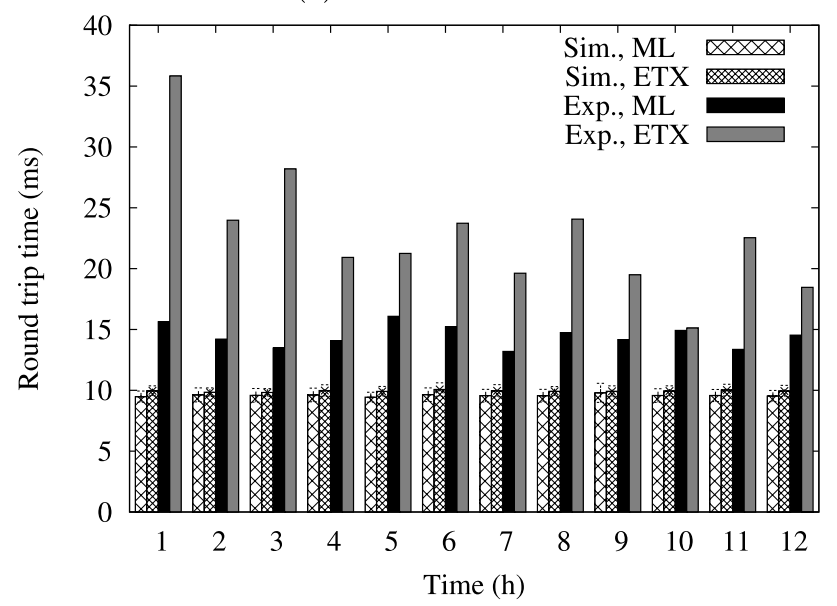

(b) Network delay.

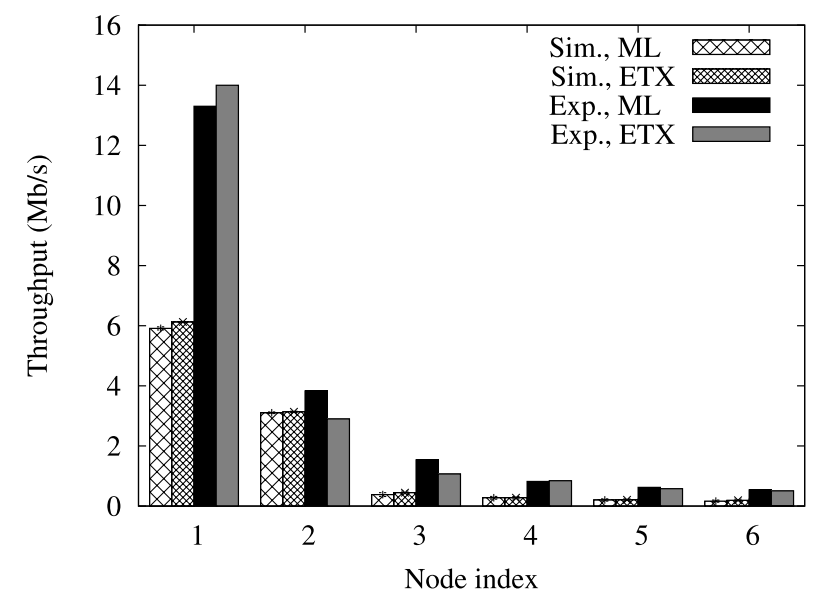

(c) Throughput.

Fig. 7 Results comparing the ML metric with the ETX metric in the Indoor Topology A

node 6). At the end of each hour, the percentage of lost packets is computed. This test allows the evaluation of the loss rate during a considerable amount of time, which includes different periods of the day. Figure 7(a) plots the loss rate at the end of each hour. This figure shows that the ETX met- ric presents higher loss rates because it tends to choose links with lower delivery rates compared with the links chosen by ML. Experiments and simulation results present similar behavior. Achieving lower loss rates is an important characteristic since many applications do not tolerate frequent packet losses [18].

\subsubsection{Network delay}

The same logs used to compute the packet loss rate were used to compute the network Round-Trip Time (RTT). The RTT metric is natively reported by the ping tool.

Results for the indoor topology A, shown in Fig. 7(b), are based on the average RTT computed for each hour of test. We observe that the average RTT with the ETX metric was always higher. Once again, we notice the consequence of ETX choosing lower quality links. Although ML tends to pick paths with a higher number of links, the links chosen provide better success probabilities than the links chosen by ETX. As a side effect, poorer links are also more unstable. This leads to a higher number of link breakages, and furthermore, to path changes. Using the $-R$ option of the ping tool, which shows the first 9 hops used by the packet, we could verify that during an 1-hour test the ETX metric switched routes more than 400 times, while the ML metric used only one route.

Here again, experimental and simulation results show similar behavior, however, the difference in simulation results is not high. These results confirm that even in a controlled environment, the ML metric outperforms ETX. Reducing RTT is another important issue in wireless networks especially considering QoS (Quality of Service) constrained applications [5].

\subsubsection{Throughput}

We perform throughput measurements using ETX and ML. We used the Iperf tool [26] in our experiments and the FTP (File Transfer Protocol) traffic generator in ns-2. In both topologies, tests were performed over reliable data transfers using the TCP protocol. We chose TCP to avoid sustained network congestion.

In the indoor topology A, 5-minute transfers were performed from node 0 to each other node. Figure 7(c) shows the results. In this plot, each node is identified by a number which gives an idea of geographical distance between the node and the gateway. The farthest node is node number 6 as seen in Fig. 5.

In this case, both metrics present similar throughput. The same result is achieved in both experimental tests and simulations. Although ML presents lower packet loss rate and RTT in our previous tests using ping based flows, under heavier traffic (such as the TCP flow of this experiment) the 
throughput obtained with the ML metric is equivalent to the throughput with ETX. Notice that under heavier traffic ML performance should be more affected than ETX because ML likely chooses longer paths. The higher the number of hops, the greater the intra-flow interference. This effect of increasing intra-flow interference could be mitigated by using an intra-flow interference aware version of TCP, such as TCPAP [14]. Nevertheless, our results show that ML performs even better in some cases, e.g., nodes $2,3,5$, and 6 .

\subsection{MLAC metric analysis}

A set of tests was performed to evaluate a few values for the additive $\lambda$ parameter defined by the MLAC metric. The topology used for these tests was the indoor topology B. Once again, the metric was implemented on the OLSR protocol.

Four different values of the parameter $\lambda$ were evaluated: $0,0.3,0.7$, and 1 . It is important to notice that when $\lambda$ is zero, the MLAC metric is identical to the ML metric.

To evaluate the performance of each of the $\lambda$ values, three different parameters were observed: the packet loss rate, the network delay, and the network throughput using the TCP protocol.

\subsubsection{Packet loss rate}

The packet loss rate tests were performed again using ping. During one day, 36 experiments of 60 seconds were performed with each value of $\lambda$. Each experiment consisted in sending 600 pings ( 1 every $100 \mathrm{~ms}$ ) of 1024 bytes. The source was a PC connected to the network gateway and the tests were repeated to every other network node.

We minimize the effects of link-quality variations swapping the different parameters in a round robin fashion. Our goal is to avoid a given measurement to profit from favorable conditions during a certain period of time. For a given value of $\lambda$, seven consecutive experiments were performed, one for each destination. After that, the $\lambda$ values are changed and another sequence of seven experiments is performed. The plot in Fig. 8(a) shows the average packet loss for each other destination node in the $X$-axis. We rerun the same experiment in our simulations.

For the intermediate values $(0.3$ and 0.7$)$ the loss rates were lower for all destinations. The higher loss rates obtained by $\lambda=1$ occurs because MLAC chooses shorter paths, as in the Hop Count metric. Increasing $\lambda$, we reduce the ML metric. Therefore, the addition of a link in the path becomes more costly. On the other hand, when the value of $\lambda$ is 0 , the problem of unitary metric persists. Therefore, the routing protocol can choose routes composed of a higher number of links. This effect is clearer in our experiments than in our simulation. Nevertheless, in our simulations, this effect becomes evident only for farther nodes, e.g., node 7.

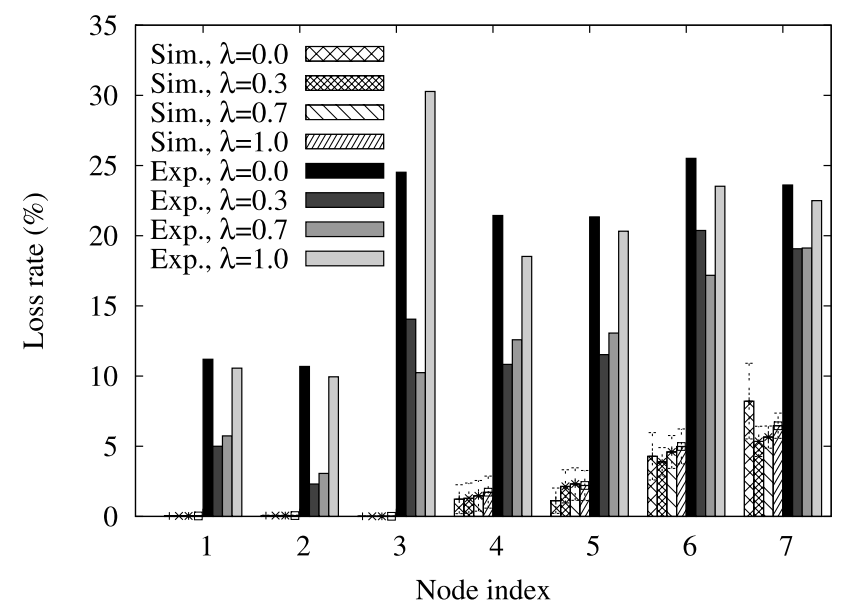

(a) Packet loss rate.

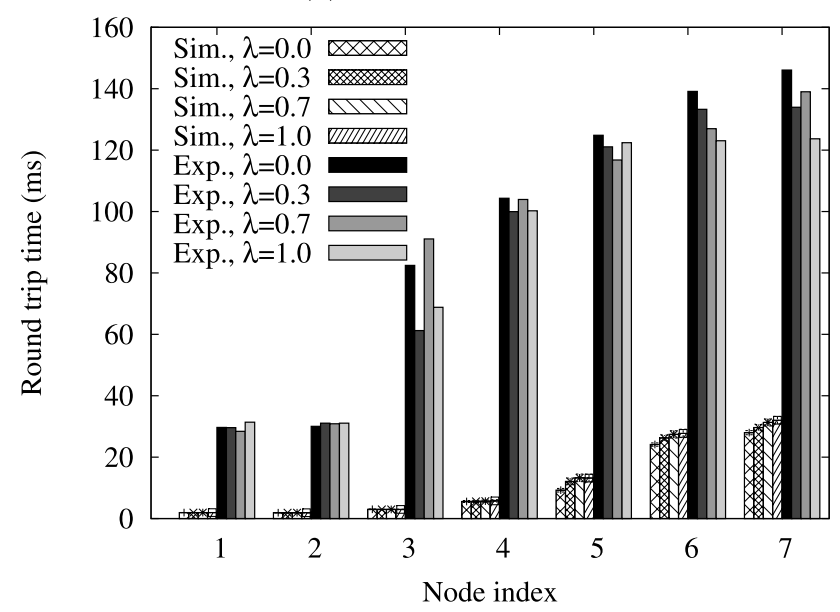

(b) Network delay.

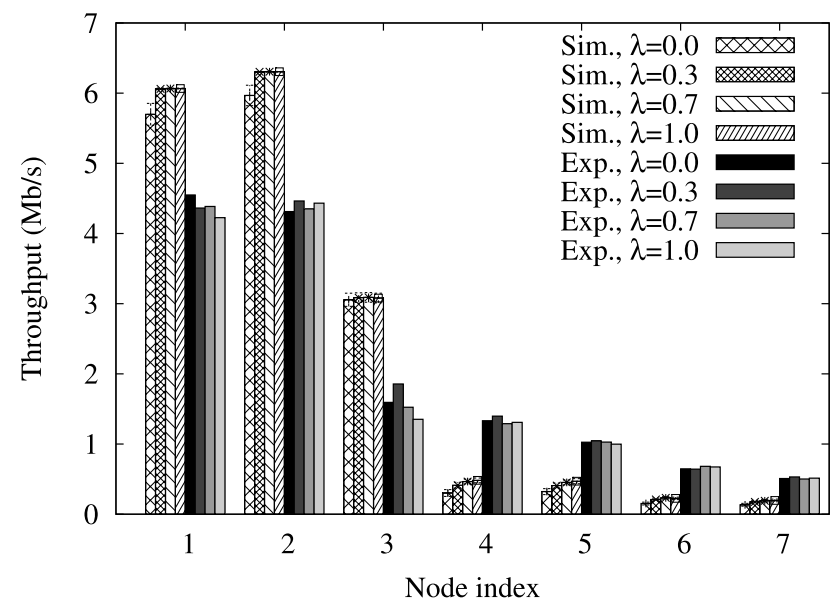

(c) Throughput.

Fig. 8 Results with the MLAC metric in the Indoor Topology B

\subsubsection{Network delay}

The RTT measurements also use the traces obtained with our ping tests. 
Figure 8(b) plots the average RTT for each destination node, varying the $\lambda$ value. The performance obtained by all metrics was very similar. Only in the results of nodes 5, 6 , and 7 , which are geographically distant from the source node (hence, there are more paths available), delay tends to be higher when using $\lambda=0$. Simulations results are similar for all $\lambda$ values, which agrees with our experiments.

\subsubsection{Throughput}

The network throughput is obtained with a script based on the iperf tool whereas the File Transfer Protocol (FTP) traffic generator in ns-2 was used in our simulations. These tests were performed similarly to the tests presented in Sects. 7.2.1 and 7.2.2. In other words, for each network node, but the gateway, 36 experiments were performed with each one of the 4 values of $\lambda$. Each experiment consisted of one reliable data transfer with the TCP protocol during 60 seconds. As with the loss rate and delay tests, these experiments were conducted in a round-robin fashion with packets of 1500 bytes.

Figure 8(c) shows the average throughput obtained on each experiment, varying the parameter $\lambda$ and the destination node. Results are very similar in both experiments and simulations. This means that in our scenario, the different values of $\lambda$ do not considerably influence the throughput obtained. This is an indirect consequence of TCP congestion control mechanism which reduces its transmission rate when packet loss increases.

\subsection{ML metric with unicast rate probing analysis}

The ETX metric collects information about each link by broadcasting probes. Broadcast transmissions are performed at the network basic rate which incurs in the overestimating problem discussed in Sect. 4. Changing this characteristic in most drivers is difficult or even impossible for users. Some particular implementations of the ETT metric tackle this problem by unicasting probes to calculate each link ETX using data transmission rates [15]. This approach, however, leads to an excessive overhead, since the same control packet needs to be sent to every neighbor individually. Moreover, unicast packets usually can be retransmitted by the link layer, which makes the measurements even more inaccurate.

In this section, we aim at evaluating the performance of a variant of ML. The proposed variant, called ML Metric with Unicast Rate Probing (MLURP), broadcast control packets at the same rate used by unicast data packets. Our ultimate goal is to evaluate the impact of estimating link conditions by broadcasting control probes at the same exact rate used for data packets. Indeed, one could argue that this proposal is not portable, because the broadcast transmission rate is a parameter not available in most wireless devices. Besides, even when available, modifying the modulation for the broadcast packets is not a trivial task. Nevertheless, in the context of this work, we aim at evaluating the impact of the inaccurate measurements on the network performance.

The evaluation of the MLURP metric was performed similarly to the MLAC tests. In MLURP, however, the data transmission rate of all routers was set to $11 \mathrm{Mb} / \mathrm{s}$. For comparison purposes, two different experiments were conducted: with the broadcast rate fixed at $11 \mathrm{Mb} / \mathrm{s}$ and at $1 \mathrm{Mb} / \mathrm{s}$. Hence, the performance of the MLURP metric was compared to the performance of the ML metric, since ML transmits probes at $1 \mathrm{Mb} / \mathrm{s}$.

\subsubsection{Packet loss rate}

The experimental analysis performed to evaluate the packet loss rate are identical to the tests described in Sect. 7.2.1.

Figure 9(a) shows the results. The packet loss rates obtained with the MLURP metric were considerably lower than with the ML metric for all nodes. This result shows that the impact of the inaccuracy in the process of inferring probabilities, as explained on Sects. 4 and 5, is indeed considerable.

\subsubsection{Network delay}

Once again, the same logs used for computing the percentage of packets lost were used in the network delay evaluation. Similar to our previous tests, the average of the RTT values reported for each ping successfully transmitted is shown in Fig. 9(b).

In terms of delay, MLURP also achieved slightly better results than ML. This is in accordance with our argument for the better performance of ML compared with ETX. Because MLURP improves link quality estimation, it can choose links with better quality. Therefore, paths chosen by MLURP are composed of more stable links with better delivery rates. The final result is the reduction of end-to-end delay even though possibly using longer paths.

\subsubsection{Throughput}

The throughput experiments performed with MLURP were conducted as explained in Sect. 7.2.3. Nevertheless, in this evaluation, only two metrics were considered.

As shown in the plot of Fig. 9(c), in terms of throughput, the MLURP metric also achieved similar results to ML metric. Similar to the results obtained in Sect. 7.1.3, we have a tradeoff between longer path lengths and network through- 


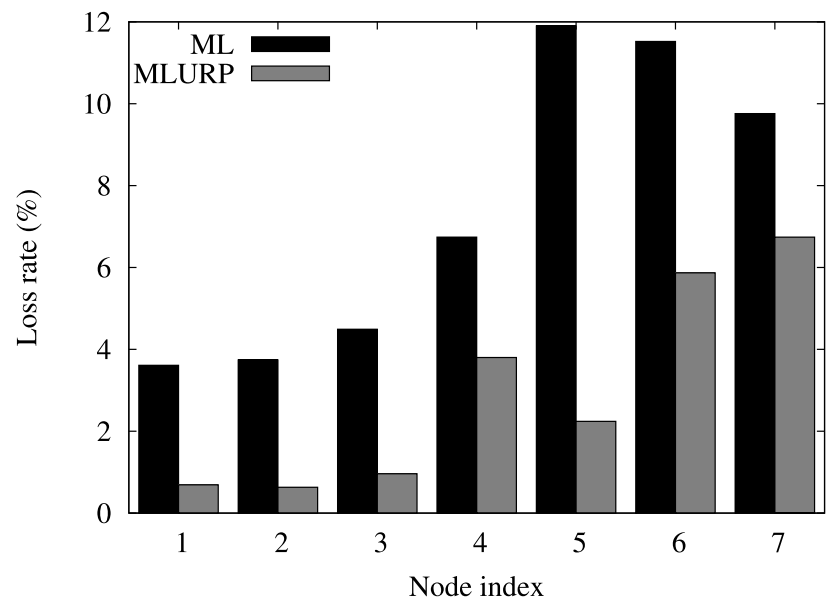

(a) Packet loss rate.

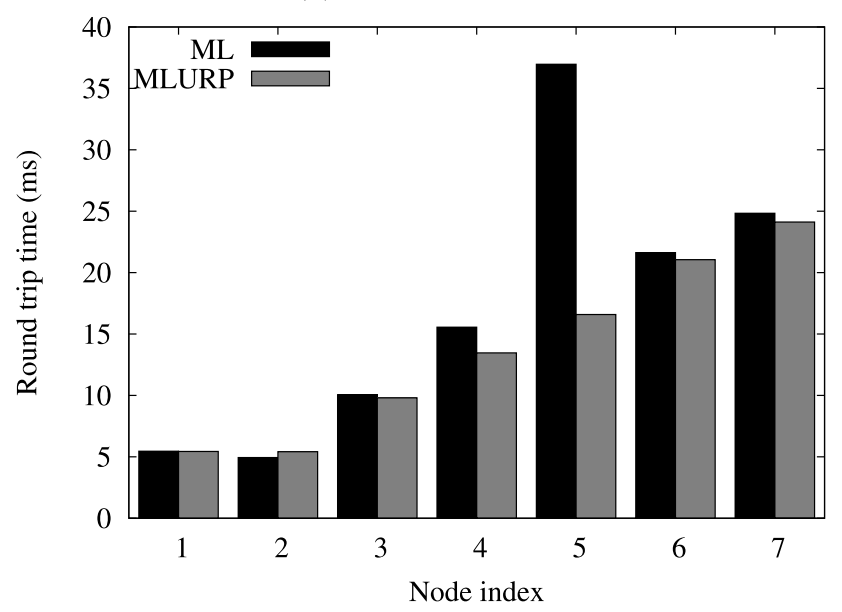

(b) Network delay.

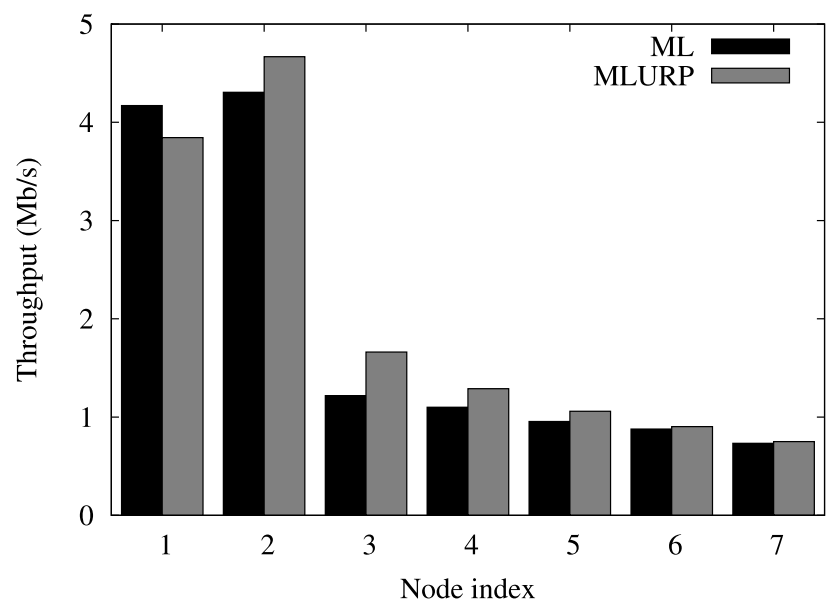

(c) Throughput.

Fig. 9 Results comparing the ML metric with the MLURP metric in the Indoor Topology B

put. In this case, however, MLURP tends to add more links than ML since MLURP estimates more accurately each link quality.

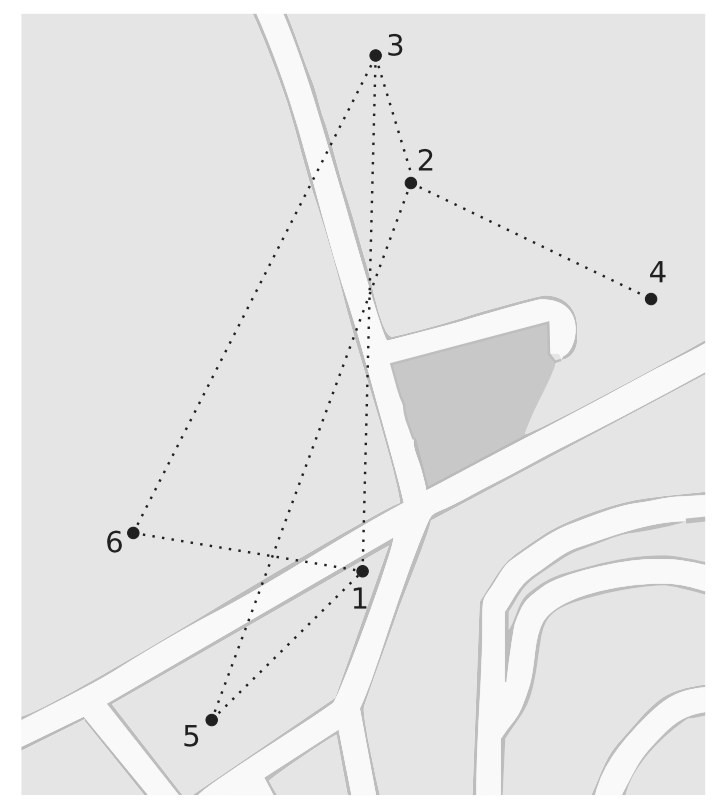

Fig. 10 Outdoor topology used in the evaluation

\section{Case study: ML metric utilization in a production network}

We implemented an access network on the surroundings of Fluminense Federal University campus. This network is used by students, faculty, and employees from our university to access the Internet. We have built an outdoor topology composed by a total of six nodes: five routers placed on the top of users' residential buildings and one gateway at the university campus. Inside each building, network connectivity is provided by an Ethernet network. Figure 10 shows the position of the nodes, as well as the links formed between them. Node 6 is the network gateway. All original antennas were replaced by $19 \mathrm{~dB}$ omni-directional antennas, but the gateway which has the original antenna replaced by a $24 \mathrm{~dB}$ directional antenna facing the user community.

The outdoor network is susceptible to great variations caused by changes in the weather, displacement of the antennas (caused by the wind), interference by other wireless networks in the neighborhood, among other factors. It is important to note that the throughput test was performed without user traffic, i.e., without concurrent network traffic. On the other hand, we also collect a number of statistics by monitoring users' traffic during network operation. In this section, we only provide experimental results.

Figures 11(a) and 11(b) plot results from 14 days of measurements with each metric. At each day, four experiments were performed. The results obtained indicate a performance gain with the ML metric in both upload and download directions, where the upload direction is the traffic from users to the Internet and download, the opposite direction. This is because ML picks paths composed of better-quality 


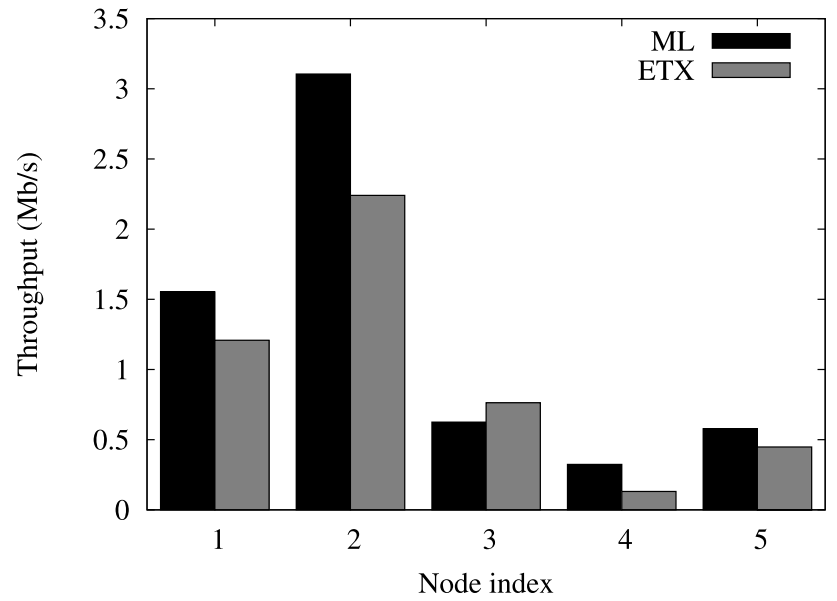

(a) Download throughput.

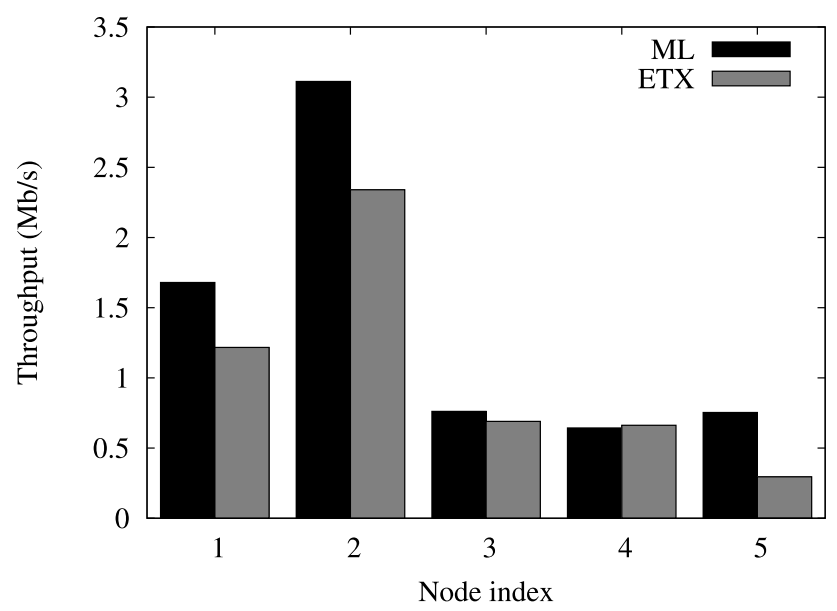

(b) Upload throughput.

Fig. 11 Throughput in the outdoor topology. Comparison between ETX and ML

links, as discussed in Sect. 7. The ML metric is effective in indoor and outdoor environments. This is an important observation which once again confirms our claim about multiplicative metrics efficiency.

From the beginning of 2006 to the end of 2007, we collected data from users' utilization. In this almost 2 years of network operation, our network evolved and more nodes were installed as seen in Fig. 12. Since the beginning over 740 Gbits were transferred to and from the top-10 bandwidth consumers in a universe of 90 users. This shows that the network was indeed actively used. Table $3^{1}$ illustrates the amount of data transferred to and from those top-10 users. We also collect statistics about the number of new connections each user established with the network. Table 4 shows the number of different days the top-10 more frequent users

\footnotetext{
${ }^{1}$ In both Tables 3 and 4, real usernames were replaced to preserve their privacy.
}

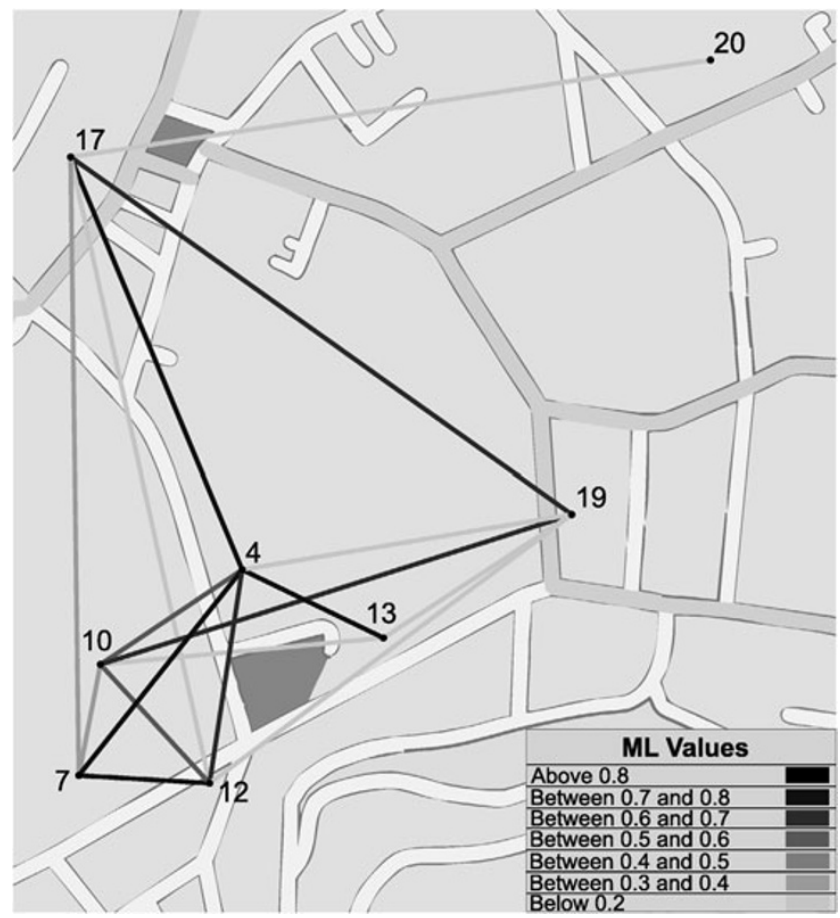

Fig. 12 Final outdoor topology

Table 3 Amount of traffic transferred to and from the top-10 bandwidth consumers in bits

\begin{tabular}{lrrr}
\hline User & $\begin{array}{l}\text { Incoming } \\
\text { traffic }\end{array}$ & $\begin{array}{l}\text { Outgoing } \\
\text { traffic }\end{array}$ & Total \\
\hline User1 & $130.2 \mathrm{G}$ & $180.4 \mathrm{G}$ & $310.6 \mathrm{G}$ \\
User2 & $78.8 \mathrm{G}$ & $133.9 \mathrm{G}$ & $212.7 \mathrm{G}$ \\
User3 & $61.1 \mathrm{G}$ & $51.8 \mathrm{G}$ & $112.9 \mathrm{G}$ \\
User4 & $19.5 \mathrm{G}$ & $5.2 \mathrm{G}$ & $24.6 \mathrm{G}$ \\
User5 & $18.7 \mathrm{G}$ & $3.1 \mathrm{G}$ & $21.8 \mathrm{G}$ \\
User6 & $17.1 \mathrm{G}$ & $838.5 \mathrm{M}$ & $17.9 \mathrm{G}$ \\
User7 & $14.1 \mathrm{G}$ & $1.8 \mathrm{G}$ & $15.9 \mathrm{G}$ \\
User8 & $2.7 \mathrm{G}$ & $8.8 \mathrm{G}$ & $11.5 \mathrm{G}$ \\
User9 & $8.8 \mathrm{G}$ & $1.2 \mathrm{G}$ & $10.0 \mathrm{G}$ \\
User10 & $5.1 \mathrm{G}$ & $1.6 \mathrm{G}$ & $6.8 \mathrm{G}$ \\
\hline
\end{tabular}

were connected to our network. It is interesting to note that the two rankings shown in Tables 3 and 4 are not the same. Therefore, the users of our network have different profiles and probably use the network for different applications.

\section{Conclusion}

With the popularization of the wireless mesh networks, there is a growing need for the development of new technologies to support such networks. The specific characteristics of this kind of network have been studied, but not yet completely 
Table 4 Different days the top-10 more frequent users were connected to the network

\begin{tabular}{ll}
\hline User & Different days connected \\
\hline User7 & 313 \\
User5 & 303 \\
User1 & 203 \\
User8 & 197 \\
User3 & 191 \\
User4 & 179 \\
User11 & 129 \\
User10 & 98 \\
User12 & 83 \\
User2 & 76 \\
\hline
\end{tabular}

explored. Thus, mesh networks have not been able to develop all its potential, in terms of performance and services. Given the diversity of projects which use these networks to promote digital inclusion, this kind of research becomes even more important.

In this work, we presented a discussion about one of the many research areas related to mesh networks: the routing metrics. They are fundamental to mesh networks, because of the need to constantly evaluate links and routes without interfering on the network performance.

A study about the main proposals in this area was performed. In special, we have analyzed the Expected Transmission Count additive metric (ETX), one of the most used metrics in mesh networks implementations and base for many other proposals in the literature. Besides this study, a multiplicative metric was analyzed. The Minimum Loss metric has the goal of minimizing the packet loss rate in an end-to-end path. Based on this metric, two variations were investigated in order to cope with other aspects of multihop wireless networks. One of these metrics, MLURP, has the objective of increasing the precision of the link quality statistics used by many metrics. Therefore, the proposed technique is applicable to other metrics, such as the ETX metric.

The tests performed on real scenarios had the objective to demonstrate the validity of the use of multiplicative metrics in certain mesh topologies. The performance results of the ML metric showed an improvement in terms of packet loss rate, route stability, network delay and, in several cases, throughput.

Another interesting result was obtained with the MLAC metric. The tests showed that by varying the additive factor $\lambda$, it is possible to obtain a performance superior to the original ML metric, what is consistent with the proposed theory.

Finally, the tests performed using the MLURP metric quantified the loss in performance caused by the imprecision in probability measures used by metrics based on this kind of statistic. Especially on the results of packet loss, the characteristic both metrics try to minimize, the MLURP metric was considerably superior to the ML metric. Although there are many obstacles for the implementation of the MLURP metric, these results show the utility of a more coherent information about link qualities.

Furthermore, a case study of the ML metric utilization in a production network was described. The results presented in this work confirm our claim on the importance of investigating multiplicative metrics. This work shows that existent metrics still present practical issues related to the gathering of link quality statistics and to their composition into a path quality that decisively affect the WMN routing performance. Hence, we argue it is clear the need for further research in WMN routing metrics.

Acknowledgements The authors would like to thank CAPES, CNPq, and FAPERJ for their support.

\section{References}

1. Akyildiz IF, Wang X, Wang W (2005) Wireless mesh networks: a survey. Comput Netw 47(4):445-487

2. Baldo N, Maguolo F, Miozzo M (2008) A new approach to simulating PHY, MAC and routing. In: International conference on performance evaluation methodologies and tools (ValueTools), pp 1-9

3. Bicket J, Aguayo D, Biswas S, Morris R (2005) Architecture and evaluation of an unplanned $802.11 \mathrm{~b}$ mesh network. In: ACM international conference on mobile computing and networking (MobiCom), pp 31-42

4. Bruno R, Conti M, Gregori E (2005) Mesh networks: commodity multihop ad hoc networks. IEEE Commun Mag 43(3):123-131

5. Campista MEM, Passos DG, Esposito PM, Moraes IM, de Albuquerque CVN, Muchaluat-Saade D, Rubinstein MG, Kosmalski Costa LHM, Duarte OCMB (2008) Routing metrics and protocols for wireless mesh networks. IEEE Netw 22(1):6-12

6. Clausen T, Jacquet P, Laouiti A, Muhlethaler P, Qayyum A, Viennot L (2001) Optimized link state routing protocol. In: IEEE international multi topic conference (INMIC), pp 62-68

7. Cordeiro W, Aguiar E, Moreira A, Abelem W, Stanton M (2007) Providing quality of service for mesh networks using link delay measurements. In: IEEE international conference on computer communications and networks (ICCCN), pp 991-996

8. Costa LHMK, Fdida S, Duarte OCMB (2002) Developing scalable protocols for three-metric QoS-routing. Comput Netw 39(6):713727

9. Crowcroft J, Wang Z (1996) Quality-of-service routing for supporting multimedia applications. IEEE J Sel Areas Commun 14(7):1228-1234

10. da Silva C, Passos D, Duarte J, Moraes I, de Albuquerque CVN (2010) Dyntun: A tool for providing multihoming support in wireless mesh networks. In: International information and telecommunication technologies symposium (I2TS) (accepted)

11. De Couto D, Aguayo D, Bicket J, Morris R (2003) A highthroughput path metric for multi-hop wireless routing. In: ACM international conference on mobile computing and networking (MobiCom), pp 134-146

12. de Paschoalino CR, Madeira ERM (2007) A scalable link quality routing protocol for multi-radio wireless mesh networks. In: IEEE international conference on computer communications and networks (ICCCN), pp 1053-1058 
13. Draves R, Padhye J, Zill B (2004) Routing in multi-radio, multihop wireless mesh networks. In: ACM international conference on mobile computing and networking (MobiCom), pp 114-128

14. ElRakabawy SM, Klemm A, Lindemann C (2005) Tcp with adaptive pacing for multihop wireless networks. In: Proceedings of the 6th ACM international symposium on mobile ad hoc networking and computing. ACM, New York, pp 288-299

15. Esposito PM, Campista MEM, Moraes IM, Rubinstein MG, Costa LHMK, Duarte OCMB (2008) Implementing the expected transmission time metric for OLSR wireless mesh networks. In: IFIP wireless days conference, pp 1-5

16. Fall K, Varadhan K The ns Manual. UC Berkeley, LBL, USC/ISI, and Xerox PARC, July 2009

17. He T, Chan S-H, Wong C-F (2008) HomeMesh: a low-cost indoor wireless mesh for home networking. IEEE Commun Mag 46(12):79-85

18. Hong D, Albuquerque C, Oliveira C, Suda T (2001) Evaluating the impact of emerging streaming media applications on TCP/IP performance. IEEE Commun Mag 39(4):76-82
19. Koksal CE, Balakrishnan $\mathrm{H}$ (2006) Quality-aware routing metrics for time-varying wireless mesh networks. IEEE J Sel Areas Commun 24(11):1984-1994

20. olsrd (2007) Accessed in http://www.olsr.org

21. OpenWrt (2008) Accessed in http://openwrt.org/

22. Parissidis G, Karaliopoulos M, Spyropoulos T, Baumann R, Plattner B (2009) Routing metrics for wireless mesh networks. In: Guide to wireless mesh networks. Springer, London, pp 199-230

23. Passos D, Teixeira D, Muchaluat-Saade D, Magalhães L, de Albuquerque CVN (2006) Mesh network performance measurements. In: International information and telecommunication technologies symposium (I2TS), pp 48-55

24. Ribeiro Torres W, D'Almeida Sanchez ML (2008) SNMP parameters for 802.11 network performance measuring. In: Ambi-Sys workshop on Software Organisation and MonIToring of Ambient Systems (SOMITAS), pp 1-7

25. Ros FJ (2005) Accessed in http://masimum.dif.um.es/um-olsr/ $\mathrm{html} /$

26. Tirumala A, Qin F, Dugan J, Ferguson J, Gibbs K (2008) Accessed in http://dast.nlanr.net/Projects/Iperf/ 